\title{
Avaliação de diferentes métodos imunoturbidimétricos para determinação de albumina urinária: impacto na classificação dos estágios da nefropatia diabética
}

\author{
Evaluation of different immunoturbidimetric methods to measure urinary albumin: \\ impact in the classification of diabetic nephropathy stages
}

Andrea Elisabet Wendland'; Mirela Jobim de Azevedo²; Jorge Luiz Gross; Joíza Lins Camargo ${ }^{4}$

unitermos
Diabetes mellitus
Nefropatia diabética
Albumina urinária
Microalbuminúria
Metodologia

\section{resumo}

Introdução: A nefropatia diabética (ND) acomete até 40\% dos pacientes diabéticos e o diagnóstico precoce pode evitar a evolução para estágios avançados. O rastreamento deve ser realizado pela medida de albumina urinária (AlbU) utilizando-se o método quantitativo sensível. Objetivo: Avaliar diferentes métodos imunoturbidimétricos de determinação de AlbU para a classificação dos estágios da ND. Material e método: A albumina foi dosada em 167 urinas (65 urinas de $24 \mathrm{~h}$ e 102 amostras casuais) por dois métodos imunoturbidimétricos: kit Aptec-BioSys, ADVIA® 1650 Bayer (AlbUAdvia) e kit MAlb Urin-Pack Bayer ${ }^{\circledR}$, Cobas Mira ${ }^{\circledR}$ Roche (AlbUCobas). AlbUCobas foi definido como método comparativo e utilizado para classificar as amostras em normoalbuminúricas (albuminúria $<30 \mathrm{mg} / 24 \mathrm{~h}$ ou $<17 \mathrm{mg} / \mathrm{l} ; \mathrm{n}=81$ ), microalbuminúricas (albuminúria $30-299 \mathrm{mg} / 24 \mathrm{~h}$ ou $17-173 \mathrm{mg} / \mathrm{l} ; \mathrm{n}=55$ ) e macroalbuminúricas (albuminúria $>300 \mathrm{mg} / 24 \mathrm{~h}$ ou $>174 \mathrm{mg} / \mathrm{l}, \mathrm{n}=31$ ). Os coeficientes de variação (CV) intra e interensaio, sensibilidade e linearidade dos métodos foram calculados. As concordâncias analítica e diagnóstica foram analisadas por regressão Deming, gráficos de Bland-Altman e por coeficiente kappa. Resultados: Os CVs intra e interensaio foram $<5 \%$ para ambos os métodos. A sensibilidade foi de $5 \mathrm{mg} / \mathrm{l}$ para ambos os métodos e a linearidade, $160 \mathrm{mg} / \mathrm{l}$ para AlbUCobas e $200 \mathrm{mg} / \mathrm{l}$ para AlbUAdvia. A concordância analítica entre os dois métodos foi adequada (média das diferenças entre os métodos = -7,68 mg/l [-0,21 - 15,2]; $r=0,989 ; p<0,001)$ e o coeficiente kappa, excelente $(0,914 ; p<0,001)$. Apenas nove amostras de urina $(5,4 \%)$ discordaram quanto à classificação pelo AlbUCobas: seis amostras microalbuminúricas foram classificadas erroneamente como normoalbuminúricas $(n=3)$ e macroalbuminúricas $(n=3)$. Três amostras normoalbuminúricas foram consideradas microalbuminúricas pelo AlbUAdvia. Os valores de albuminúria dessas amostras encontravam-se no limite superior dos pontos de corte diagnósticos dos respectivos estágios de ND. Conclusão: Os métodos imunoturbidimétricos analisados podem ser intercambiáveis sem ocasionar mudanças significativas na classificação dos diferentes estágios da ND.

abstract

Background: Diabetic nephropathy (DN) affects up to $40 \%$ of diabetic patients and must be screened by the measurement of urinary albumin with a sensitive quantitative method. Objective: To evaluate the impact of different immunoturbidimetric methods to measure albuminuria in the classification of DN stages. Material and method: Albumin was measured in 167 urine samples (65 $24 \mathrm{~h}$ samples and 102 casual samples) by two immunoturbidimetric methods: Aptec BioSys, ADVIA® 1650 Bayer (AlbUAdvia) and Malb Urin-Pack Bayer ${ }^{\circ}$, Cobas Mira $\AA$ Roche (AlbUCobas). AlbUCobas was defined as the comparative method used to classify the samples in: normoalbuminuric (albuminuria $<30 \mathrm{mg} / 24 \mathrm{~h}$ or $<17 \mathrm{mg} / \mathrm{l} ; \mathrm{n}=81$ ), microalbuminuric (albuminuria 30-299 mg/24 hor 17-173 mg/l; $\mathrm{n}=55$ ) and macroalbuminuric (albuminuria $>300 \mathrm{mg} / 24 \mathrm{~h}$ or $>174 \mathrm{mg} / \mathrm{l} ; \mathrm{n}=31)$. The intra and interassay coefficients of variation (CVs), sensitivity and linearity of each method were calculated and the analytical and diagnostic agreements were analyzed by Deming's regression and Bland-Altman plots and by Kappa coefficient, respectively. Results: The intra and interassay CVs were $<5 \%$ for both methods. The sensitivity was $5 \mathrm{mg} / \mathrm{l}$ for both methods and the linearity was 160 $\mathrm{mg} / \mathrm{I}$ for AlbUCobas and $200 \mathrm{mg} / \mathrm{l}$ for AlbUAdvia. The analytical agreement between the two methods was satisfactory (mean differences between methods $=-7.68 \mathrm{mg} / \mathrm{l}(-0.21-15.2) ; \mathrm{r}=0.989 ; \mathrm{p}<0.001$ ) and the kappa coefficient $(0,914 ; \mathrm{p}<0,001)$ was excellent. Only nine urine samples $(5,4 \%)$ were in disagreement with AlbUCobas classification: six microalbuminuric samples were misclassified as normoalbuminuric $(\mathrm{n}=3)$ and macroalbuminuric $(\mathrm{n}=3)$ by AlbUAdvia. Three normoalbuminuric samples were misclassified as microalbuminuric by AlbUAdvia. The albumin values of these samples were in the highest diagnostic adopted cut-off point for each DN stage. Conclusion: The immunoturbidimetric methods analyzed may be interchangeable, without significant misclassification of the different stages of DN. key words

Diabetes mellitus

Diabetic nephropathy

Urinary albumin

Microalbuminuria

Methodology

\footnotetext{
1. Farmacêutica-bioquímica da Unidade de Bioquímica e Imunoensaios do Serviço de Patologia Clínica do Hospital de Clínicas de Porto Alegre (HCPA).

2. Professora associada do Departamento de Medicina Interna da Faculdade de Medicina da Universidade Federal do Rio Grande do Sul (UFRCS); médica do Serviço de Endocrinologia do HCPA.

3. Professor-titular do Departamento de Medicina Interna da Faculdade de Medicina da UFRCS; chefe do Serviço de Endocrinologia do HCPA.

4. Professora do Programa de Pós-graduação em Ciências Médicas: Endocrinologia; chefe da Unidade de Bioquímica e Imunoensaios do Serviço de Patologia Clínica do HCPA.
} 


\section{Introdução}

O diabetes mellitus (DM) é uma síndrome metabólica crônica, caracterizada por hiperglicemia, que resulta de defeitos na secreção e/ou ação da insulina ${ }^{(1)}$. A hiperglicemia crônica está associada a complicações micro e macrovasculares ${ }^{(11-13)}$. As complicações crônicas do DM podem causar, a longo prazo, falência de vários órgãos, entre eles os rins, levando ao aparecimento de nefropatia diabética (ND) $)^{(5,8,9)}$.

A ND acomete mais de um terço dos pacientes e é a causa mais comum de ingresso em programas de hemodiálise ${ }^{(3)}$. Os diferentes estágios clínicos da ND estão demonstrados na Tabela 1. A evidência mais precoce da ND é o aparecimento de valores de albumina na urina não detectados por métodos tradicionais de medida da proteinúria (30-300 mg/24 h ou 20-200 $\mu \mathrm{g} / \mathrm{min}$ ou $17-173 \mathrm{mg} / \mathrm{l}^{(5,7)}$, definidos como microalbuminúria ou nefropatia incipiente. Sem intervenção específica, aproximadamente $80 \%$ dos pacientes com DM tipo 1 (DM1) microalbuminúricos progridem para macroalbuminúria ou nefropatia clínica ( $\geq 300 \mathrm{mg} / 24 \mathrm{~h}$ ou $\geq 200 \mu \mathrm{g} / \mathrm{min}$ ou $\geq 174 \mathrm{mg} / \mathrm{l}$ ) num período de 10 a 15 anos $^{(5,8,9)}$. Em pacientes com DM tipo 2 (DM2), 20\% a 40\% dos pacientes com microalbuminúria progridem para a nefropatia clínica, e, após 20 anos, cerca de $20 \%$ desenvolvem doença renal terminal(5,8). Além disso, a presença de microalbuminúria em pacientes com DM (tipos 1 e 2) já é um indicativo de risco aumentado para doença cardiovascular ${ }^{(5,8,9)}$.

Estudos como o Diabetes Control and Complications Trial (DCCT) e o United Kingdom Prospective Diabetes Study (UKPDS) demonstraram que terapia intensificada do DM com o objetivo de manter os níveis de glicose e o controle da pressão arterial $(\mathrm{PA})$ o mais próximo do normal pode reduzir significativamente o risco de desenvolvimento e progressão da ND em pacientes com $\mathrm{DM}^{(12,13)}$. Portanto, a detecção precoce da nefropatia tem importantes implicações práticas no sentido da adoção de medidas preventivas e terapêuticas específicas, visando a melhoria da qualidade de vida e modificação da evolução da ND. Para isso, a determinação quantitativa de albumina urinária (AlbU) por método sensível é necessária para realizar o rastreamento e a determinação do estágio da ND em que o paciente se encontra $(5,6,9,10)$.

A microalbuminúria, por raramente ocorrer em pacientes com DM1 com curta duração de doença, deve ter seu rastreamento iniciado cinco anos após o diagnóstico de DM. Já em pacientes com DM2, devido à dificuldade em determinar o início da doença, o rastreamento para a ND deve ser realizado no momento do diagnóstico do DM. Após o rastreamento inicial, e na ausência de microalbuminúria, um teste para avaliar sua presença deve ser realizado anualmente ${ }^{(5,8,9)}$.

O rastreamento para microalbuminúria pode ser realizado com dosagens de albumina em amostras de urina casuais, urinas de $24 \mathrm{~h}$ ou com tempo cronometrado $(5-7,70,14)$, assim como pela razão albumina/creatinina, em amostra de urina casual, com resultados expressos em mg de albumi$\mathrm{na} / \mathrm{g}$ de creatinina $^{(7)}$ (Tabela 1).

Ensaios específicos são necessários para detectar microalbuminúria, pois os ensaios convencionais para detecção de proteínas urinárias não são suficientemente sensíveis para detectar os níveis reduzidos de albumina na urina neste estágio da $\mathrm{ND}^{(5,10)}$. A técnica mais utilizada é a imunoturbidimetria (IT), que se baseia na formação de um complexo antígeno-anticorpo, utilizando um anticorpo específico para a albumina humana ${ }^{(4)}$.

As mudanças de kits ou métodos muitas vezes se fazem necessárias na rotina do laboratório clínico. É imprescindível

\section{Tabela 1 Valores de albumina utilizados para o diagnóstico de nefropatia diabética}

\begin{tabular}{lcc}
\hline Categoria & Urina $24 \mathrm{~h}^{*}$ & Amostra casual \\
Normoalbuminúria & $<20 \mu \mathrm{m} / \mathrm{min}$ & $<17 \mathrm{mg} / /^{* *}$ \\
& $<30 \mathrm{mg} / 24 \mathrm{~h}$ & $<30 \mathrm{mg} / \mathrm{g}$ creatinina \\
Nefropatia incipiente ou microalbuminúria & 20 a $199 \mu \mathrm{\mu g} / \mathrm{min}$ & $17-173 \mathrm{mg} / /^{* *}$ \\
& 30 a $299 \mathrm{mg} / 24 \mathrm{~h}$ & $30-300 \mathrm{mg} / \mathrm{g} \mathrm{creatinina}$ \\
Nefropatia clínica ou macroalbuminúria & $\geq 200 \mu \mathrm{g} / \mathrm{min}$ & $\geq 174 \mathrm{mg} / /^{* *}$ \\
& $\geq 300 \mathrm{mg} / 24 \mathrm{~h}$ & $>300 \mathrm{mg} / \mathrm{g}$ creatinina \\
\hline
\end{tabular}

*Adaptado de American Diabetes Association, 2004.

**Adaptado de Zelmanowitz T et al., 1997.

\#Adaptado de KDOQI Clinical Practice Guidelines and Clinical Practice Recommendations for Diabetes and Chronic Kidney Disease, 2007. 
que se proceda à validação adequada desses novos métodos para que erros ou diferenças significativas nos resultados dos exames laboratoriais sejam evitados, garantindo a correta classificação da ND e, conseqüentemente, o manejo clínico e terapêutico adequado dos pacientes.

O presente estudo tem por objetivo avaliar o impacto da utilização de diferentes métodos imunoturbidimétricos para determinação de AlbU na classificação dos estágios da ND.

\section{Material e métodos}

Neste estudo de teste diagnóstico foram avaliadas 167 amostras de urina de pacientes diabéticos atendidos no Serviço de Endocrinologia do Hospital de Clínicas de Porto Alegre (HCPA), entre as quais 65 de urina de $24 \mathrm{~h}$ e 102 de urinas casuais. Para a inclusão das amostras foram selecionados pacientes de ambos os sexos e portadores de DM1 ou DM2. As dosagens foram realizadas na Unidade de Bioquímica e Imunoensaios do Serviço de Patologia Clínica do HCPA durante o período de novembro de 2004 a janeiro de 2005.

A AlbU foi determinada por IT, por dois imunoensaios diferentes: kit Aptec-BioSys, equipamento ADVIA ${ }^{\circledR} 1650$ Bayer (AlbUAdvia) e kit MAlb Urin-Pack Bayer ${ }^{\circledR}$, equipamento Cobas Mira ${ }^{\circledR}$ Roche (AlbUCobas). AlbUCobas foi definido como método comparativo e utilizado para classificar as amostras como normoalbuminúricas (albuminúria $<30 \mathrm{mg} / 24$ h ou $<17 \mathrm{mg} / \mathrm{l} \mathrm{em}$ amostra casual de urina), microalbuminúricas (albuminúria 30-299 mg/24 h ou 17-173 mg/l) e macroalbuminúricas (albuminúria $>300 \mathrm{mg} / 24 \mathrm{~h}$ ou $>174 \mathrm{mg} / \mathrm{l}$ ). A escolha do método comparativo deveu-se ao seu uso na rotina do Laboratório de Patologia Clínica do HCPA desde o ano de 1996, período em que foi extensivamente estudado e padronizado ${ }^{(3,6,14,15)}$. As amostras foram conservadas entre $2^{\circ}$ e $8^{\circ} \mathrm{C}$ até o momento da dosagem, que ocorreu no máximo até cinco dias após a coleta, simultaneamente nos dois equipamentos. As dosagens foram realizadas nos diferentes equipamentos no decorrer do mesmo dia.

Para garantir o controle de qualidade interno das medidas de albuminúria, durante a determinação de todas as urinas analisadas foram concomitantemente dosados um pool de urinas com valor de albumina determinado pelo laboratório $(69,2 \mathrm{mg} / \mathrm{l})$ pelo método AlbUCobas e um controle comercial de valor conhecido $(232 \mathrm{mg} / \mathrm{l})$.

$\mathrm{O} C \mathrm{~V}$ intra-ensaio foi determinado por duas amostras de urina com valores distintos de albumina (22,2 e 241,2 mg/l), as quais foram dosadas 10 vezes numa mesma corrida. Para a determinação do CV interensaio foram medidas, durante 10 dias consecutivos, amostras do pool de urinas e do controle comercial em duplicata. A sensibilidade e a linearidade da técnica foram determinadas por meio de diluições sucessivas de uma mesma amostra de urina. Para a sensibilidade foi utilizada uma amostra selecionada aleatoriamente com o valor de albumina de $80 \mathrm{mg} / \mathrm{l}$, e para a linearidade uma amostra com valor de $1.200 \mathrm{mg} / \mathrm{l}$.

Para o controle do eventual efeito pró-zona nos imunoensaios para albumina, a proteína urinária total (PTU) foi dosada por ensaio colorimétrico (vermelho de pirogalol, Labtest, $A D V I A^{\circledast} 1650$ Bayer) previamente à dosagem de albuminúria em todas as amostras. Essa medida estimou a contribuição da albumina na concentração de PTU em cada amostra. Considerou-se que, em média, 50\% da proteína total são constituídos de albumina (na faixa de micro e macroalbuminúria) $)^{(15)}$. A partir dessa estimativa foi realizada uma diluição da amostra, conforme mostra a Tabela 2, permitindo a medida da albuminúria dentro da faixa de medição ou sensibilidade fornecida pelo fabricante de cada ensaio. As urinas com PTU $<300$ mg/l não foram previamente diluídas.

\begin{tabular}{cc|} 
Tabela 2 & $\begin{array}{l}\text { Diluições das amostras de urina } \\
\text { utilizadas para controle do efeito } \\
\text { pró-zona }\end{array}$ \\
\hline Proteína urinária total (mg/l) & Diluição \\
\hline Até 300 & sem diluição \\
$310-500$ & $1: 2$ \\
$510-1.000$ & $1: 5$ \\
$1.010-2.000$ & $1: 10$ \\
$2.010-3.000$ & $1: 20$ \\
$3.010-4.000$ & $1: 30$ \\
\hline
\end{tabular}

\section{Análise estatística}

A comparação analítica dos métodos foi feita por meio dos gráficos de Bland-Altman ${ }^{(2)}$ e da correlação de Pearson. O coeficiente kappa foi utilizado para avaliar a concordância diagnóstica entre os métodos ( $k a p p a=0$ define não-concordância, e kappa $=1$ define concordância total). As variáveis foram descritas como mediana (intervalo) ou média \pm desvio padrão (DP). Foi adotado um nível de significância de 0,05 . As análises foram realizadas utilizando-se o SPSS 14.0 (SPSS, Chicago, IL). 


\section{Resultados}

O desempenho analítico dos métodos estudados está demonstrado na Tabela 3. Os CVs intra e interensaios foram $<5 \%$ nas diferentes faixas de albuminúria, utilizando-se o pool de urinas in house e o controle comercial durante 10 dias.

Os resultados obtidos foram semelhantes nas duas técnicas, com excelente correlação $(r=0,989, p<0,001)$ e adequada concordância (média das diferenças entre as duas técnicas $=-7,68 \mathrm{mg} / \mathrm{l}[-0,21-15,2])$ (Figura e Tabela 3).

Das 167 amostras de urina estudadas, 31 foram classificadas como macroalbuminúricas, 55 como microalbuminúricas e 81 como normoalbuminúricas pelo método de referência (AlbUCobas). Quando analisadas pelo método em avaliação (AlbUAdvia), 34 amostras foram classificadas como macroalbuminúricas, 52 como microalbuminúricas e 81 como normoalbuminúricas (Tabela 4). A concordância diagnóstica entre os dois métodos, em relação à classificação dos pacientes nos diferentes estágios da nefropatia, foi excelente $(96,64 \%)$, correspondendo a um coeficiente kappa de 0,914 $(p<0,001)$. Do total das amostras analisadas, apenas nove
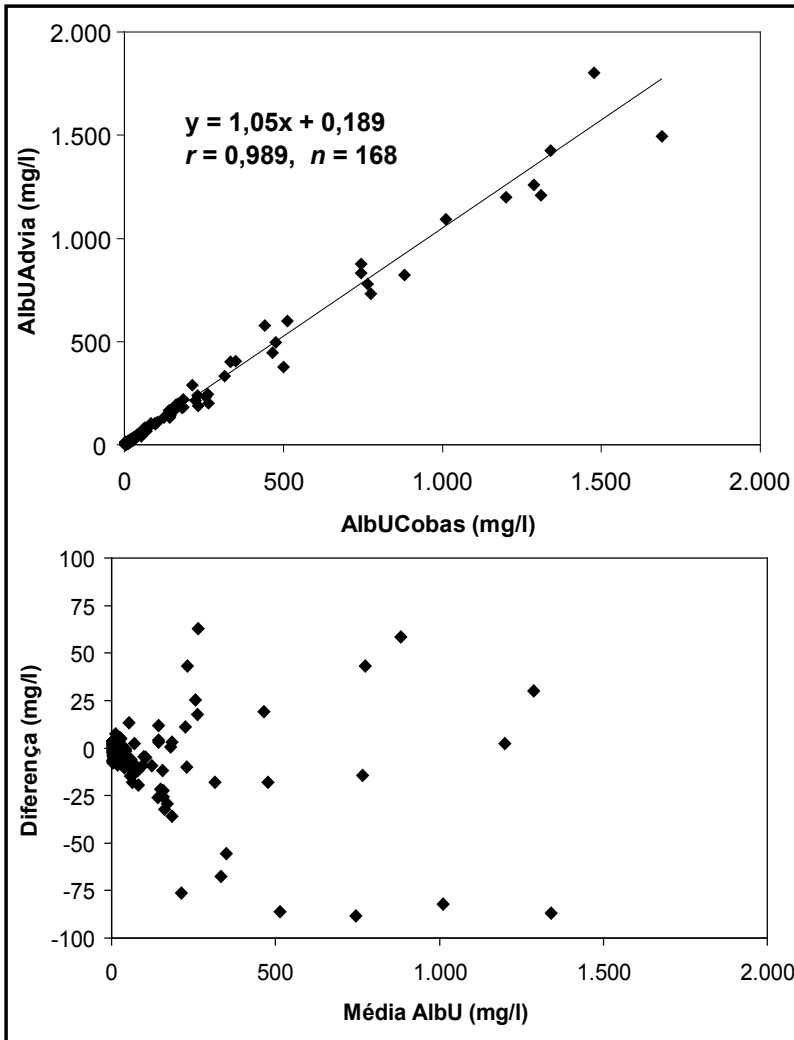

Figura - Concordância analítica entre os métodos: $x=$ AlbuCobas e $y=$ AlbUAdvia a linha contínua representa $x=y$

\section{Tabela 3 Desempenho analítico dos imunoensaios para medidas de albumina urinária}

\begin{tabular}{lcc}
\hline & AlbUCobas & AlbUAdvia \\
Albuminúria $(\mathrm{mg} / \mathrm{l})^{*}$ & 20,3 & 20,4 \\
& $(5-1.691)$ & $(5-2036)$ \\
CV intra-ensaio (\%) & 4,5 & 4 \\
CV interensaio (\%) & 4,6 & 4,1 \\
Linearidade (mg/l) & 160 & 200 \\
Sensibilidade (mg/l) & 5 & 5 \\
Coeficiente de correlação & \multicolumn{2}{c}{0,989} \\
Média da diferenças entre os métodos (mg/l; 95\% IC) & \multicolumn{2}{c}{$-7,68(-0,21-15,2)$} \\
\hline
\end{tabular}

*Mediana (intervalo)

IC: intervalo de confiança.

Concordância diagnóstica entre os dois imunoensaios para determinação de albuminúria nos

Tabela 4 estágios de nefropatia diabética*

\begin{tabular}{cccccc}
\hline & & \multicolumn{3}{c}{ AlbU Cobas } & \\
\multirow{3}{*}{ AlbU Advia } & Normo & Micro & Macro & Índice kappa \\
& Normo & 79 & 3 & - & \\
& Micro3 & 3 & 49 & - & 0,914 \\
& Macro & - & 3 & 30 & \\
\hline
\end{tabular}

*Em número de amostras de urina.

Normo = normoalbuminúria; micro = microalbuminúria; macro = macroalbuminúria . 
$(5,36 \%)$ discordaram do estágio de ND determinado pelo método de referência (AlbUCobas): seis microalbuminúricas foram classificadas erroneamente como normoalbuminúricas ( $n=3)$ e macroalbuminúricas $(n=3)$; três normoalbuminúricas foram consideradas microalbuminúricas pelo AlbUAdvia. Os valores de albuminúria dessas amostras encontravam-se no limite superior dos pontos de corte diagnósticos dos respectivos estágios de ND. Os valores individuais de albuminúria dessas amostras estão demonstrados na Tabela 5.

\section{Discussão}

A estimativa adequada da acurácia de uma técnica de teste diagnóstico deve incluir, além da análise de seu desempenho analítico, a do impacto no diagnóstico clínico dos pacientes.

No presente estudo foi avaliado o impacto da mudança de imunoensaio utilizado para a dosagem de albumina urinária na classificação dos pacientes com ND. Demonstrouse que os dois métodos utilizados apresentaram excelente concordância analítica e diagnóstica em relação aos valores absolutos nos diferentes estágios da ND: normo, micro e macroalbuminúria. Segundo Sacks et al.(10), o CV analítico dos métodos para dosagem de microalbuminúria deve ser menor que $15 \%$. Os métodos em estudo apresentaram valores de CV bem inferiores em todas as faixas de valores analisados: CV intra-ensaio $=4,5 \%$ e $4 \%$ e CV interensaio $=4,6 \%$ e $4,1 \%$ para AlbUCobas e AlbUAdvia, respectivamente.

Do total de amostras estudadas, apenas 5,36\% não apresentaram a mesma classificação quando avaliadas pelos dois métodos. Os valores de albuminúria dessas amostras encontravam-se no limite superior dos pontos de corte diagnósticos utilizados (Tabela 5). Esse fato explica por que essas amostras urinárias foram classificadas no mesmo estágio da ND, apesar de se observar uma diferença entre os dois métodos analisando-se apenas os valores absolutos. O diagnóstico definitivo de um determinado estágio da ND deve ser sempre confirmado em uma segunda medida de albuminúria, em ocasião diferente $\mathrm{e}^{(5,10)}$.

Cuidados metodológicos foram observados para que fosse mantido o padrão de qualidade das medidas de albumina. Uma vez que a albumina é estável em urinas não tratadas, as amostras podem ser armazenadas a $4^{\circ} \mathrm{C}$ por pelo menos uma semana ${ }^{(10)}$. Neste estudo, após conservação em temperatura adequada, as amostras foram medidas em tempo hábil (até cinco dias). Ademais, as amostras foram dosadas nos dois equipamentos no mesmo dia, em diferentes horários. Assim, é pouco provável que alterações decorrentes do processo de armazenagem, como tempo e temperatura, tenham influenciado os resultados.

Em todas as amostras foi dosada a proteína urinária total, como uma forma de controle interno da qualidade da técnica, para estimar a quantidade de albumina esperada em cada amostra, considerando que a albumina corresponde a aproximadamente $50 \%$ da proteína total(15). Esse procedimento teve como principal objetivo controlar um possível efeito pró-zona em amostras com concentração de albumina superior ao limite de linearidade do método, permitindo que as diluições necessárias fossem feitas antes da dosagem da albuminúria. Além de garantir qualidade e aumentar a acurácia dos resultados, esse procedimento evita repetições da medida de albumina urinária em amostras com concentrações elevadas e proporciona uma economia para o laboratório de

Valores individuais de albuminúria ( $\mathrm{mg} / \mathrm{l}$ ou $\mathrm{mg} / 24 \mathrm{~h}$ ) das nove amostras discordantes quanto à

Tabela 5 classificação do estágio de nefropatia diabética pelos métodos AlbUCobas e AlbUAdvia

\begin{tabular}{cccccc}
\hline \multirow{2}{*}{ Amostra 1 } & Cobas mg/l & Advia mg/l & & Cobas mg/24 h & Advia mg/24 h \\
& 13,5 & 17,4 & Amostra 5 & 26,8 & 30,8 \\
Amostra 2 & Normo & Micro & & Normo & Micro \\
& 19,9 & 14,5 & Amostra 6 & 24,5 & 37 \\
Amostra 3 & Micro & Normo & & Normo & Micro \\
& 18,7 & 12,8 & Amostra 7 & 36,3 & 20,4 \\
Amostra 4 & Micro & Normo & & Micro & Normo \\
& 169,4 & 198,8 & Amostra 8 & 294 & 340,6 \\
& Micro & Macro & & Micro & Macro \\
& - & - & Amostra 9 & 271,5 & 311,2 \\
\hline
\end{tabular}

Normo = normoalbuminúria; micro = microalbuminúria; macro = macroalbuminúria . 
análises clínicas. O custo para uma dosagem de albumina por IT é aproximadamente $R \$ 2,00$, enquanto que a dosagem de proteínas totais por turbidimetria é cerca de $\mathrm{R} \$ 0,50$.

\section{Conclusão}

A correta classificação do estágio da ND é fator decisivo para o prognóstico e o tratamento dos pacientes com
$\mathrm{DM}^{(5)}$. Portanto sugere-se que os laboratórios clínicos, ao adotar um novo método para medida de albumina urinária, realizem um protocolo de avaliação de teste diagnóstico semelhante ao do presente estudo.

Em conclusão, os métodos imunoturbidimétricos analisados podem ser intercambiáveis sem ocasionar mudanças significativas na classificação dos diferentes estágios da ND.

\section{Referências}

1. AMERICAN DIABETES ASSOCIATION. Diagnosis and classification of diabetes mellitus. Diabetes Care, v. 7 , suppl. 1, p. S5-S10, 2004.

2. BLAND, J. M.; ALTMAN, D. G. Statistical methods for assessing agreement between two methods of clinical measurement. Lancet, v. 8, p. 307-10, 1986.

3. BRUNO, R. M.; GROSS, J. L. Prognostic factors in Brazilian diabetic patients starting dialysis: a 3,6 year follow-up study. J Diabetes Complications, v. 14, n. 5, p. 26671, 2000.

4. COLLEGE OF AMERICAN PATHOLOGISTS. Surveys 2006 U-B Urine Chemistry. Northfield, IL, 2006.

5. GROSS, J. L. et al. Diabetic nephropathy: diagnosis, prevention, and treatment. Diabetes Care, v. 28, p. 164-76, 2005.

6. INCERTI, J. et al. Evaluation of tests for microalbuminuria screening in patients with diabetes. Nephrol Dial Transplant, v. 20, p. 2402-7, 2005.

7. KDOQI. Clinical Practice Guidelines and Clinical Practice Recommendations for Diabetes and Chronic Kidney Disease - Guideline 1: Screening and Diagnosis of Diabetic Kidney Disease. Am J Kidney Diseases, v. 49, n. 2, suppl. 2, p. S42-S61, 2007.

8. MOLITCH, M. E. et al. Nephropathy in diabetes. Diabetes Care, v.27, suppl. 1, p. S79-83, 2004.

9. REMUZZI, G. et al. Nephropathy in patients with type 2 diabetes. N Engl J Med, v. 346, n. 15, p. 1145-51, 2002.
10. SACKS, D. B. et al. Guidelines and recommendations for laboratory analysis in the diagnosis and management of Diabetes Mellitus. Clin Chem, v. 48, n. 3, p. 436-72, 2002.

11. SCHEFFEL, R. S. et al. Prevalência de complicações micro e macrovasculares e de seus fatores de risco em pacientes com diabetes melito tipo 2 em atendimento ambulatorial. Rev Assoc Med Bras, v. 50, n. 3, p. 2637, 2004.

12.THE DIABETES CONTROL AND COMPLICATIONS TRIAL RESEARCH GROUP. The effect of intensive treatment of diabetes on the development and progression of long-term complications in insulin-dependent diabetes mellitus. N Engl J Med, v. 329, p. 977-86, 1993.

13. UK PROSPECTIVE DIABETES STUDY GROUP. Intensive blood-glucose control with sulphonylureas or insulin compared with conventional treatment and risk of complications in patients with type 2 diabetes (UKPDS 33). Lancet, v. 352, p. 837-53, 1998.

14. ZELMANOVITZ, T. et al. The receiver operating characteristics curve in the evaluation of a random urine specimen as a screening test for diabetic nephropathy. Diabetes Care, v. 20, p. 516-9, 1997.

15. ZELMANOVITZ, T. et al. Proteinuria is still useful for the screening and diagnosis of overt diabetic nephropathy. Diabetes Care, v. 21, p. 1076-9, 1998. 